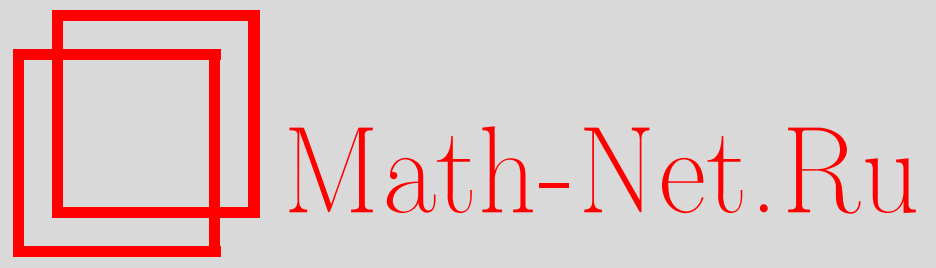

Ж. М. Р. Гама, В. В. Юринский, Показательное неравенство для всплесковой оценки плотности, Теория вероятн. и ее примен., 2006, том 51, выпуск 4, 674-690

DOI: https://doi.org/10.4213/tvp19

Использование Общероссийского математического портала MathNet.Ru подразумевает, что вы прочитали и согласны с пользовательским соглашением

http://www . mathnet.ru/rus/agreement

Параметры загрузки:

IP : 54.162 .85 .209

26 апреля 2023 г., 15:19:41

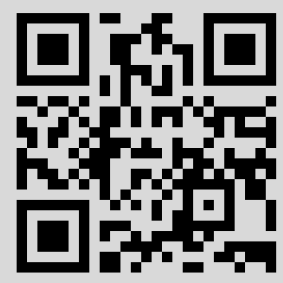


(c) $2006 \Gamma$.

\author{
ГАМА Ж. М.Р.*, ЮРИНСКИЙ В. В.*
}

\title{
ПОКАЗАТЕЛЬНОЕ НЕРАВЕНСТВО ДЛЯ ВСПЛЕСКОВОЙ ОЦЕНКИ ПЛОТНОСТИ ${ }^{1)}$
}

Статья посвящена выводу показательной оценки для хвоста распределения $L^{p}$-нормы невязки между неизвестной одномерной плотностью распределения и ее «нелинейной» всплесковой (вэйвлетной) оценкой, использующей срезки коэффициентов.

Предполагается, что в рассматриваемых кратномасштабных разложениях масштабирующая функция и всплеск имеют компактные носители. Полученная в заметке показательная оценка сродни известному неравенству С. Н. Бернштейна для сумм независимых случайных величин. Она дополняет известные оценки для среднего интегрального риска. Вывод оценки использует почти полную независимость эмпирических приближений к коэффициентам всплескового разложения для базисных всплесков одного и того же уровня разрешения в случае, когда носители их разделены достаточным расстоянием.

Ключевые слова и фразы: оценивание плотности, большие уклонения, всплесковые оценки, пороговая срезка коэффициентов, показательные неравенства.

\section{1. Введение}

Ниже рассматривается задача оценки неизвестной плотности вероятности по выборке, состоящей из независимых одинаково распределенных наблюдений с этой плотностью. Хорошо известно, что всплесковые оценки плотности (ВОП) оказываются чрезвычайно эффективными в этой ситуации. ${ }^{2)}$

Сушествует обширная литература, посвященная асимптотическому анализу эффективности ВОП с помощью характеристик, выражаемых

* Departamento de Matemática, Universidade da Beira Interior, Rua Marquês d'Ávila e Bolama, 6201-001 Covilhã, Portugal; e-mail: jgama@mat.ubi.pt, yurinsky@ubi.pt

1) Работа выполнена при поддержке фонда Fundação para a Ciência e a Tecnologia (Portugal) через посредство Centro de Matemática da Universidade da Beira Interior, Sub-Projecto DECONT.

2) Терминология, относящаяся к всплескам (вэйвлетам), следует [5]. 
моментами подходящих норм невязки между оценкой и искомой плотностью. Этот подход позволил установить оптимальные скорости сходимости оценок, равномерные при выборе неизвестной плотности из классов плотностей, удовлетворяющих специфическим условиям регулярности, и привел к достаточно глубокому пониманию эффектов, вызываюших радикальное уменьшение интегрального риска ВОП при исключении из линейной проекционной оценки $[2$, гл. 12$]$ «несущественных» компонент, для которых эмпирические коэффициенты кратномасштабного разложения не достигают предписанных пороговых значений (см., например, [8], [12]).

Несколько иной подход был использован в [1.1], [9]. В этих работах свойства ВОП анализируются в ситуациях, где ставится цель восстановить индивидуальную плотность распределения, изменяюшуюся с увеличением объема выборки и сочетающую гладкую «фоновую» составляющую и растущее число менее регулярных элементов, существенно влияющих на высокочастотную часть кратномасштабного разложения плотности (в [11] такими элементами являются скачки, а в [9] - сужающиеся при увеличении выборки колоколообразные пики искомой плотности). В цитированных работах анализ эффективности основывался на вычислении математического ожидания интеграла квадрата невязки [11] или ее математического ожидания в точках, где находятся пики, возмущающие гладкую фоновую плотность [9]. Этот анализ дал интересную дополнительную информацию о характере сходимости нелинейной ВОП к нерегулярной искомой плотности.

Главный результат настоящей заметки приведен ниже в теореме 1.1 и относится к стандартной «пороговой» ВОП, использующей так называемую «жесткую срезку» коэффициентов ${ }^{3)}$, т.е. полное исключение из кратномасштабного разложения исходной проекционной оценки компонент, у которых модули коэффициентов не достигают некоторых пороговых значений. При довольно слабых ограничениях на неизвестную плотность распределения $f$ эта теорема доставляет экспоненциальное неравенство для «хвоста» распределения погрешности ВОП $\|\tilde{f}-f\|_{p}$, где $\|\cdot\|_{p}$ - норма в пространстве $L^{p}(\mathbf{R}), p \geqslant 2$. Оно сродни классическому неравенству С. Н. Бернштейна [1], адаптированному к большим уклонениям сумм независимых случайных векторов $[16, \S 3.3]$, хотя сушественно нелинейная природа сжатия данных при преобразовании проекционной оценки в ВОП, использующую срезки коэффициентов, мешает непосредственному применению этого последнего. При доказательстве теоремы 1.1 основным инструментом является рандомизация объема выборки с тем, чтобы полнее использовать практическую независимость коэффициентов кратномасштабного разложения проекционной

3) «Hard thresholding». 
оценки плотности, которая служит основой при построении «нелинейной» пороговой ВОП - этот прием использовался ранее, например, в работах [4] об оценивании функции регрессии двумерного распределения и [15] о ядерных оценках двумерных плотностей.

Мы сосредоточиваем внимание преимущественно на случайных составляющих невязки между ВОП и искомой плотностью, для которых вероятности больших уклонений определяются некоторыми грубыми характеристиками распределения и всплескового базиса. Влияние более тонких свойств плотности и кратномасштабного базиса на математическое ожидание и моменты пороговой ВОП исследовано с достаточной полнотой (см. [8], [12] и приведенную в этих работах библиографию, а также [11], [9]). По этой причине мы отделяем в теореме 1.1 случайные составляющие погрешности оценки от детерминированных и ограничиваемся немногими замечаниями относительно поведения этих последних.

Основной результат. ВОП, рассматриваемые ниже, основываются на кратномасштабном разложении [7], [14], [5] неизвестной плотности в $L^{2}(\mathbf{R})$

$$
f=\sum_{k} \alpha_{J_{*} k} \phi_{J_{*} k}+\sum_{j>J_{*}} \sum_{k} \beta_{j k} \psi_{j k},
$$

где коэффициентами служат математические ожидания $\alpha_{J_{*} k}=\mathbf{E} \phi_{J_{*} k}(X)$ и $\beta_{j k}=\mathbf{E} \psi_{j k}(X)$ (здесь $X-$ случайная величина с плотностью $f$, а сокращение $\sum_{k}$ заменяет, как и всюду ниже, выражение $\left.\sum_{k \in \mathbf{Z}}\right)$. Кратномасштабное разложение (1.1) порождается масштабирующей функцией (шапочкой) $\phi$ и всплеском (вэйвлетом) $\psi$, используя ортонормальный базис, состоящий из функций

$\phi_{J_{*} k}(x)=\lambda_{J_{*}}^{-1 / 2} \phi\left(\lambda_{J_{*}}^{-1}\left(x-x_{J_{*}}\right)\right), \quad \psi_{j k}(x)=\lambda_{j}^{-1 / 2} \psi\left(\lambda_{j}^{-1}\left(x-x_{j k}\right)\right)$,

где $\lambda_{j}=\left(A 2^{j}\right)^{-1}$ и $x_{j k}=k \lambda_{j}$ при $j \geqslant J_{*}$.

Оценка плотности строится по выборке объема $n$, состоящей из независимых одинаково распределенных случайных величин $X_{j}$ с плотностью распределения $f$ из (1.1). Эта выборка определяет средние

$$
\begin{aligned}
a_{J_{*} k} & =a_{J_{*} k}\left(X_{1}, \ldots, X_{n}\right)=\overline{\phi_{J_{*} k}(X)}=\frac{1}{n}\left(\phi_{J_{*} k}\left(X_{1}\right)+\cdots+\phi_{J_{*} k}\left(X_{n}\right)\right), \\
b_{j k} & =b_{j k}\left(X_{1}, \ldots, X_{n}\right)=\overline{\psi_{j k}(X)}=\frac{1}{n}\left(\psi_{j k}\left(X_{1}\right)+\cdots+\psi_{j k}\left(X_{n}\right)\right) .
\end{aligned}
$$

Приведенные выше средние являются несмешенными оценками соответствующих коэффициентов в (1.1). Линейная ВОП $[12, \S 10.2]$ (или, в другой терминологии, проекционная оценка плотности, использующая ортонормальную систему $\left(\phi_{J_{\star}, k}, \psi_{j, \ell}\right)$, см. [6] и [2, гл. 12]), задается разложением (1.1) с конечным числом уровней, $J_{*}<j \leqslant J^{*}$, в котором $\alpha_{J_{*} k}$ и $\beta_{j k}$ заменены соответственно на $a_{J_{*} k}$ и $b_{j k}$. 
Пороговая ВОП с жесткой срезкой $\tilde{f}$ получается из линейной оценки исключением компонент, у которых модули коэффициентов $b_{j k}$ из (1.3) не достигают некоторого заданного порогового значения $\delta_{j}=\delta_{j}(n)>0$, $j>J_{*}$ :

$$
\tilde{f}(x)=\sum_{k} a_{J_{*} k} \phi_{J_{*} k}(x)+\sum_{J_{*}<j \leqslant J^{*}} \sum_{k} \widetilde{b}_{j k} \psi_{j k}(x),
$$

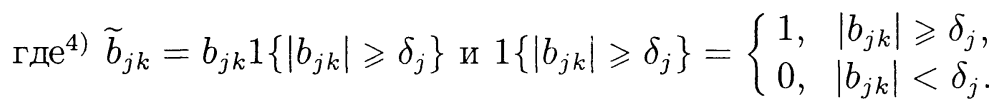

Мы будем сравнивать оценку (1.4) со случайным элементом $L^{2}(\mathbf{R}) \cap L^{p}(\mathbf{R}), p \geqslant 2$, который не является оценкой плотности и получается исключением из линейной ВОП компонент с малыми математическими ожиданиями коэффициентов $\beta_{j k}$ (ниже $\gamma>0$ - параметр, который может варьироваться по выбору):

$$
\hat{f}_{n}(x)=\sum_{k} a_{J_{*} k} \phi_{J_{*}}(x)+\sum_{J_{*}<j \leqslant J^{*}} \sum_{k} b_{j k} 1\left\{\left|\beta_{j k}\right| \geqslant(1+2 \gamma) \delta_{j}\right\} \psi_{j k}(x) .
$$

Невязка между искомой плотностью и пороговой ВОП может быть представлена в виде

$$
\tilde{f}-f=(\tilde{f}-\hat{f})+(\hat{f}-\mathbf{E} \hat{f})+\widehat{B}_{1}+\widehat{B}_{J^{*}}, \quad \hat{f}=\hat{f}_{n},
$$

с неслучайными последними слагаемыми

$$
\begin{aligned}
\widehat{B}_{1} & =-\sum_{J_{*}<j \leqslant J^{*}} \sum_{k} \beta_{j k} 1\left\{\left|\beta_{j k}\right|<(1+2 \gamma) \delta_{j}\right\} \psi_{j k} \\
\widehat{B}_{J^{*}} & =\sum_{j>J^{*}} \sum_{k} \beta_{j k} \psi_{j k}=f-\sum_{k} \alpha_{J_{*} k} \phi_{J_{*} k}(x)-\sum_{J_{*}<j \leqslant J^{*}} \sum_{k} \beta_{j k} \psi_{j k}(x) .
\end{aligned}
$$

Мы будем оценивать вероятности больших уклонений для случайных слагаемых правой части (1.6).

В дальнейшем масштабируюшая функция и всплески в (1.4) предполагаются ограниченными и имеющими компактные носители: существуют константы $\Phi_{1}>0$ и $K \in \mathbf{N}$ такие, что

$$
\begin{array}{ll}
\|\phi\|_{\infty}=\sup _{x}|\phi(x)| \leqslant \Phi_{1}, & \operatorname{supp}(\phi) \cap \operatorname{supp}\left(\phi \circ T_{K}\right)=\varnothing, \\
\|\psi\|_{\infty}=\sup _{x}|\psi(x)| \leqslant \Phi_{1}, & \operatorname{supp}(\psi) \cap \operatorname{supp}\left(\psi \circ T_{K}\right)=\varnothing,
\end{array}
$$

где $g \circ T_{K}(x)=g(x+K)$ обозначает функцию, полученную из $g$ сдвигом.

Искомая плотность распределения удовлетворяет условию

$$
\exists q_{*} \geqslant p \geqslant 2 \quad\|f\|_{q_{*}}=\left(\int_{\mathbf{R}} f^{q_{*}}(x) d x\right)^{1 / q_{*}}<\infty .
$$

\footnotetext{
4) Индикаторы множеств обозначаются $1_{A}(x), 1\{x \in A\}$ и т.д.
} 
Ниже мы уделяем основное внимание существенно нелинейной случайной составляющей невязки $\tilde{f}-\hat{f}$. Для линейных комбинаций всплесков одного уровня разрешения мы будем использовать известные неравенства между $L^{p}(\mathbf{R})$-нормой функции и $l^{p}$-нормой $|\eta|_{p}=\left(\sum_{k}\left|\eta_{k}\right|^{p}\right)^{1 / p}$ последовательности ее коэффициентов (см. [8, лемма 1] или $[14$, т. 1]): при $p \geqslant 1$

$$
\begin{aligned}
& c_{1} \lambda_{J^{*}}^{-(1 / 2-1 / p)}|\eta|_{p} \leqslant\left\|\sum_{k} \eta_{k} \phi_{J_{*} k}\right\|_{p} \leqslant c_{2} \lambda_{J^{*}}^{-(1 / 2-1 / p)}|\eta|_{p}, \\
& c_{1} \lambda_{j}^{-(1 / 2-1 / p)}|\eta|_{p} \leqslant\left\|\sum_{k} \eta_{k} \psi_{j k}\right\|_{p} \leqslant c_{2} \lambda_{j}^{-(1 / 2-1 / p)}|\eta|_{p},
\end{aligned}
$$

где константы $c_{i}$ зависят от $p$ и выбора всплескового базиса.

3 а м е ч а н и е 1.1. Вследствие неравенства (1.9) первый неслучайный член в правой части (1.6) допускает оценку

$$
\left\|\widehat{B}_{1}\right\|_{p} \leqslant c_{2} \mathbf{B}(\gamma, \delta), \quad \mathbf{B}(\gamma, \delta)=\sum_{J_{*}<j \leqslant J^{*}} \lambda_{j}^{-(1 / 2-1 / p)} \mathbf{b}_{j}\left(\gamma, \delta_{j}\right),
$$

где

$$
\mathbf{b}_{j}\left(\gamma, \delta_{j}\right)=\left(\sum_{k}\left|\beta_{j k}\right|^{p} 1\left\{\left|\beta_{j k}\right| \leqslant(1+2 \gamma) \delta_{j}\right\}\right)^{1 / p} .
$$

Мы будем использовать кроме введенных выше еще обозначения

$$
\mathbf{B}^{+}=\sum_{J_{*}<j \leqslant J^{*}} \lambda_{j}^{-(1 / 2-1 / p)} \mathbf{b}_{j}^{+}, \quad \mathbf{b}_{j}^{+}=\left(\sum_{k}\left|\beta_{j k}\right|^{p}\right)^{1 / p} .
$$

Основным результатом статьи является следующая теорема.

Теорема 1.1. Предположим, что $p \geqslant 2$ и выполнены условия (1.7), (1.8). Если для некоторьих $\varkappa, \mathbf{v}^{*}, \overline{\mathbf{v}}^{*}>0$ и любого челого $j \in\left(J_{*}, J^{*}\right]$ выполнень неравенства

$$
\begin{aligned}
& \overline{\mathbf{v}}_{j}=\lambda_{j} \max _{0 \leqslant s<K} \sum_{k} \mathbf{E} \psi_{j(s+K k)}^{2}(X) \leqslant \overline{\mathbf{v}}^{*}, \\
& \mathbf{v}_{j}=\sup _{k} \mathbf{E} \psi_{j k}^{2}(X) \leqslant \mathbf{v}^{*}, \quad \frac{\delta_{j}}{\delta_{J^{*}}} \geqslant \varkappa,
\end{aligned}
$$

u

$$
\frac{\Phi_{1} \varkappa}{4 \mathbf{v}^{*}} \gamma \delta_{J^{*}} \lambda_{J^{*}}^{-1 / 2} \leqslant \ln 2
$$

где параметр $\gamma$ из (1.5) таков, что $0<\gamma<\frac{1}{4}$, то вероятность

$$
\mathbf{p}_{\varepsilon}=\mathbf{P}\left\{\|\tilde{f}-\hat{f}\|_{p} \geqslant c_{2}\left(\varepsilon+\frac{2}{\gamma \sqrt{n}} \mathbf{B}^{+}+(1+4 \gamma) \mathbf{B}(\gamma, \delta)\right)\right\},
$$

определенная с использованием постоянной $c_{2}$ из неравенства (1.9), допускает оченку

$\forall \varepsilon>0 \quad \mathbf{p}_{\varepsilon} \leqslant \frac{8}{3} \exp \left\{-\frac{\varkappa \gamma^{2} \delta_{J^{*}} \varepsilon n \lambda_{J^{*}}^{1 / 2-1 / p}}{4 K^{1 / p} \chi(p) \mathbf{v}^{*}}\left(1-\frac{8 K^{1 / p} \chi(p) \overline{\mathbf{v}}^{*}}{\gamma \varepsilon \lambda_{J^{*}}^{3 / 2-1 / p} \sqrt{n}} \mu_{n}\right)\right\}$, 
¿əe

$$
\begin{array}{r}
\mu_{n}=\frac{1}{\varkappa \gamma \delta_{J^{*} \sqrt{n}}} \exp \left\{-\frac{\left(\varkappa \gamma \delta_{\left.J^{*} \sqrt{n}\right)^{2}}\right\},}{8 \mathbf{v}^{*}}\right\} \\
\chi(p)= \begin{cases}\frac{1-2^{-(1 / 2-1 / p)}}{1-2^{-\left(J^{*}-J_{*}\right)(1 / 2-1 / p)},} & p>2, \\
J^{*}-J_{*}, & p=2 .\end{cases}
\end{array}
$$

3 а м е ч а н и е 1.2. Если выполнены условия (1.7) и (1.8), то можно положить

$$
\overline{\mathbf{v}}^{*}=\Phi_{1}^{2}, \quad \mathbf{v}^{*}=\Phi_{1}^{2 / q_{*}} \lambda_{J^{*}}^{-1 / q_{*}}\|f\|_{q_{*}}
$$

$\mathrm{c}^{*}=\|f\|_{\infty}$ в предположении, что плотность ограничена.

В предположениях замечания 1.2 первое неравенство в (1.13) получается применением неравенства Гёльдера к интегралу в равенстве $\mathbf{E} \psi_{j k}^{2}\left(X_{\ell}\right)=\int \psi_{j k}^{2}(x) f(x) d x$. Второе неравенство в (1.13) верно из-за того, что носители функций $\psi_{j(s+K k)}^{2}, k \in \mathrm{Z}$, не имеют общих точек, а потому $\sum_{k} \psi_{j(s+K k)}^{2}\left(X_{\ell}\right) \leqslant \Phi_{1}^{2} \lambda_{j}^{-1}$.

3 а м е ч а н и е 1.3. Величина $\mu_{n}$ из (1.15) стремится к нулю при $n \rightarrow \infty$ не медленнее $n^{-A}$ при любом выборе $A>0$, если только $\gamma \delta_{J^{*}} \sqrt{n} \geqslant$ $T \sqrt{\mathbf{v}^{*} \ln n}$ для достаточно большого $T, T \geqslant T_{0}(\gamma, \varkappa, A)$.

Доказательство теоремы 1.1 завершается в разделе 4. В техническом отношении основу его составляют вычисления п. 3.1, основанные на рандомизации объема выборки с целью полнее использовать слабую зависимость между «далекими» коэффициентами $\widetilde{b}_{j k}$ одного уровня разрешения $T \geqslant T_{0}(\gamma, \varkappa)$ (более ранние примеры использования рандомизации со сходной целью можно найти в [15], [4]).

Вторая случайная составляющая невязки в разложении (1.6) представляется суммой одинаково распределенных независимых случайных векторов из пространства $L^{p}$ и допускает показательную оценку, аналогичную классическому неравенству Бернштейна (доказательство ее приведено в п. 2.1). Ниже $\hat{f}_{1}(x)-$ случайный элемент, определенный формулой (1.6) с объемом выборки $n=1$.

Теорема 1.2. Если $p \geqslant 2$, а число $\mathbf{V}_{\gamma \delta}$ вылбрано так, что $\mathbf{E}\left\|\hat{f}_{1}\right\|_{p}^{2} \leqslant$ $\mathbf{V}_{\gamma \delta}, m_{O}$

$$
\begin{aligned}
\forall t>0 \quad \mathbf{P} & \left\{\|\hat{f}-\mathbf{E} \hat{f}\|_{p} \geqslant\left(t+\tau_{0}\right)\left(\frac{\mathbf{V}_{\gamma \delta}}{n}\right)^{1 / 2}\right\} \\
& \leqslant 2 \exp \left\{-\frac{1}{2} t^{2}\left(1+C_{1}^{*} K^{1 / p} \Phi_{1} t \rho_{n}\right)^{-1}\right\},
\end{aligned}
$$

где $\rho_{n}=\left(\lambda_{J^{*}}^{(1-1 / p)} \sqrt{n \mathbf{V}_{\gamma \delta}}\right)^{-1}$, а положительные постояннье $C_{1}^{*}, \tau_{0}$ зависят только от р (см. ниже (2.6) и (2.7)). 
Для иллюстрации эффективности оценок теоремы 1.1 применим их в ситуациях, где известна оптимальная скорость сходимости пороговой BOП.

Предположим, что неизвестная плотность распределения принадлежит классу Бесова $\widetilde{B}(s, r, q), s \in(m, m+1]$, т.е. ее носитель содержится в интервале конечной длины $2 L$ и

$$
\|f\|_{s, r, q}=\|f\|_{r}+\left\|f^{(m)}\right\|_{r}+\left(\int_{0}^{\infty} t^{-(1+q(s-m))} \sup _{|h| \leqslant 1}\left\|\Delta_{h t}^{2} f^{(m)}\right\|_{r}^{q} d t\right)^{1 / q} \leqslant F_{s, r, q}
$$

где $1 \leqslant r, q \leqslant \infty, m \in \mathbf{Z}_{+}$и $s r>1$, что гарантирует ограниченность плотности, а потому условие (1.8) выполнено с $q_{*}=\infty$.

(a) Если погрешность оценки измеряется $L^{2}$-нормой невязки и $r=2$, то удовлетворено неравенство $2=r>2 /(2 s+1)$, и, тем самым, рассматривается регулярный случай (в терминологии $[12, \S 10.4]$ ). Оптимальная скорость сходимости достигается для ВОП, у которых минимальный используемый уровень разрешения удовлетворяет условию $\lambda_{J_{*}}^{-1} \sim n^{1 /(2 s+1)}$, и имеет порядок $\left(\mathbf{E}\|\tilde{f}-f\|_{2}^{2}\right)^{1 / 2} \simeq n^{-s /(2 s+1)}$. Мы будем сравнивать верхнюю оценку теоремы 1.1 для случая переменного порогового значения $\delta_{j}=D \sqrt{j / n}$, где $D>0$ постоянно, с почти оптимальной верхней оценкой риска [12, теорема 10.4] для ВОП, использующей срезку с тем же пороговым значением:

$$
\left(\mathbf{E}\|\tilde{f}-f\|_{2}^{2}\right)^{1 / 2} \leqslant C^{*} \mathbf{e}(n), \quad \mathbf{e}(n)=n^{-s /(2 s+1)}(\ln n)^{\beta}, \quad \beta>0 .
$$

Предположим, что $J^{*}-J_{*} \leqslant \alpha \log _{2} n$, где число $\alpha>0$ мало. $\mathrm{B}$ этом случае условия (1.13) выполняются с $\varkappa, \mathbf{v}^{*}$ и $\overline{\mathbf{v}}^{*}$, не зависяшими от $n$. Условие (1.14) выполнено, поскольку $\lambda_{J^{*}}^{-1 / 2} \leqslant \lambda_{J_{*}}^{-1 / 2} n^{\alpha}$, а потому бесконечно мала последовательность $\delta_{J^{*}} \lambda_{J^{*}}^{-1 / 2} \leqslant C(1 /(2 s+1)+$ $\alpha)(\ln n)^{1 / 2} n^{-1 / 2+1 /(2 s+1)+\alpha}$. Выбирая достаточно большим параметр $D$, можно добиться, чтобы величина $\mu_{n}$ из (1.15) сходилась к нулю быстрее любой заданной отрицательной степени $n$ при $n \rightarrow \infty$. Рассмотрим вероятность появления невязки с нормой больше $\varepsilon=\varepsilon(n)=t \mathbf{e}(n)$, т.е. почти того же порядка, что оптимальная скорость сходимости. При этом выборе масштаба невязки

$$
\begin{aligned}
\delta_{J^{*}} \varepsilon \lambda_{J^{*}}^{1 / 2-1 / 2} n\left(J^{*}-J_{*}\right)^{-1} & \sim C t n^{1-1 / 2-s /(2 s+1)}(\log n)^{1 / 2+\beta-1} \\
& =C t n^{1 /(4 s+2)}(\ln n)^{\beta-1 / 2} \longrightarrow \infty
\end{aligned}
$$

и $\mathbf{p}_{\varepsilon}(\varepsilon) \leqslant \frac{8}{3} \exp \left\{-C^{\prime} t n^{1 /(4 s+2)}(\ln n)^{\beta-1 / 2}\right\}$, что указывает на пренебрежимость вклада составляющей $\|\tilde{f}-\hat{f}\|_{2}$ в погрешность рассматриваемой BOП.

(б) Предположим теперь, что погрешность оценки плотности оценивается $L^{p}$-нормой в так называемом «разреженном» случае, когда 
$1 / s<r<p /(2 s+1)$ и, с необходимостью, $p>2$. Оптимальная скорость сходимости в этом случае есть [12, теоремы 10.3 и 10.4$]$

$$
\mathbf{e}(n)=\left(\mathbf{E}\|\tilde{f}-f\|_{p}^{p}\right)^{1 / p} \simeq\left(\frac{1}{n} \ln n\right)^{\alpha_{2}}, \quad \alpha_{2}=\frac{s-1 / r+1 / p}{2 s+1-2 / r} .
$$

Она отвечает выбору порогового значения срезки коэффициентов $\delta_{j}=$ $D \sqrt{j / n} \sim D\left(n^{-1} \ln n\right)^{1 / 2}$ при крайних уровнях разрешения $\lambda_{J_{*}}^{-1} \sim$ $\left(n(\ln n)^{\dot{p} / r-1}\right)^{\alpha_{2} / s}$ и $\lambda_{J^{*}}^{-1} \sim(n / \ln n)^{\alpha_{2} / s^{\prime}}$, где $s^{\prime}=s-1 / r+1 / p$.

Если выбранный масштаб погрешности ВОП задан равенством $\varepsilon=t \mathbf{e}(n)$, то при указанном выше выборе остальных параметров выполняется асимптотическое соотношение $\delta_{J} \widehat{\varepsilon} n \sim C t \ln n$ при $n \rightarrow \infty$, а оценка теоремы 1.1 ведет себя как

$$
\mathbf{p}_{\varepsilon} \leqslant \nu_{1} \exp \left\{-\nu_{2} t \ln n\right\}, \quad \nu_{1}, \nu_{2}>0 .
$$

Как и выше, последовательность $\mu_{n}$ в (1.15) сходится к нулю быстрее любой отрицательной степени $n$, если $D$ достаточно велико, причем условие $(1.14)$ выполнено, поскольку $\delta_{J^{*}} \lambda_{J^{*}}^{-1 / 2} \sim\left(n^{-1} \ln n\right)^{\left(1-\alpha_{2} / s^{\prime}\right) / 2}$ и $\left(1-\alpha_{2} / s^{\prime}\right) / 2=(s-1 / r) /(2(s-1 / r)+1)>0$.

\section{2. Разделение составляющих невязки}

Мы пользуемся стандартными обозначениями уровней разрешения и состветствующих операторов проектирования в схеме кратномасштабного анализа, основанной на функциях $(\phi, \psi)$, обозначая $H_{J^{*}}^{\gamma \delta}$ подпространство $L^{2}(\mathbf{R})$, которое содержит случайный элемент $\hat{f}$, определенный в (1.5):

$$
\begin{aligned}
V_{J}=\mathscr{K}_{j} L^{2}(\mathbf{R})=\{h: h= & \sum_{k} \eta_{k}^{0} \phi_{J_{*} k} \\
& \left.+\sum_{J_{*}<j \leqslant J} \sum_{k} \eta_{j k} \psi_{j k}\right\}, \quad J_{*} \leqslant J \leqslant J^{*}, \\
W_{j}=\left(\mathscr{K}_{j}-\mathscr{K}_{j-1}\right) L^{2}(\mathbf{R})=\left\{h: h=\sum_{k} \eta_{j k} \psi_{j k}\right\}, \quad j>J_{*}, & \\
H_{J^{*}}^{\gamma \delta}=\mathscr{K}_{J^{*}}^{\gamma \delta} V_{J^{*}}=\{h: h= & \left.\sum_{J_{*}<j \leqslant J^{*}} \sum_{k} \eta_{j k} 1\left\{\left|\beta_{j k}\right|>(1+2 \gamma) \delta_{j}\right\} \psi_{j k}\right\} .
\end{aligned}
$$

Величины $\beta_{j k}$ в определении $H_{J^{*}}^{\gamma \delta}$ суть коэффициенты разложения (1.1) искомой плотности. Подпространство $H_{J^{*}}^{\gamma \delta}$ конечномерно; то же заключение верно в отношении $V_{J^{*}}$, если плотность $f$ имеет компактный носитель.

2.1. Линейное приближение к пороговой ВОП. Условие (1.8) обеспечивает возможность представления плотности $f$ кратномасштабным разложением (1.1). Пороговая ВОП (1.4) и случайный вектор $\hat{f}$ 
из (1.5) получаются проектированием из среднего

$$
\overline{\boldsymbol{\xi}} \equiv \frac{1}{n}\left(\boldsymbol{\xi}_{1}+\cdots+\boldsymbol{\xi}_{n}\right)=\sum_{k} a_{J_{*} k} \phi_{J_{*} k}+\sum_{J_{*}<j \leqslant J^{*}} \sum_{k} b_{j k} \psi_{j k} \in V_{J^{*}}
$$

независимых одинаково распределенных случайных векторов

$$
\boldsymbol{\xi}_{\ell}=\boldsymbol{\xi}\left(X_{\ell}\right) \stackrel{\text { def }}{=} \sum_{k} \phi_{J_{*} k}\left(X_{\ell}\right) \phi_{J_{*} k}+\sum_{J_{*}<j \leqslant J^{*}} \sum_{k} \psi_{j k}\left(X_{\ell}\right) \psi_{j k} \in V_{J^{*}}
$$

Математическое ожидание этого последнего - проекция в $L^{2}(\mathbf{R})$ плотности $f$ на подпространство $V_{J^{*}}$ :

$$
\mathbf{E} \overline{\boldsymbol{\xi}}=\sum_{k} \alpha_{J_{*} k} \phi_{J_{*} k}+\sum_{J_{*}<j \leqslant J^{*}} \sum_{k} \beta_{j k} \psi_{j k}=\mathscr{K}_{J^{*}} f
$$

Обозначим

$$
\begin{aligned}
\xi_{\ell}^{\left(J_{*}\right)}=\left(\phi_{j k}\left(X_{\ell}\right)\right), & \mathbf{E} \xi_{\ell}^{\left(J_{*}\right)}=\left(\alpha_{j k}=\mathbf{E} \phi_{J_{*} k}\left(X_{\ell}\right)\right), \\
\xi_{\ell}^{(j)}=\left(\psi_{j k}\left(X_{\ell}\right)\right), & \mathbf{E} \xi_{\ell}^{(j)}=\left(\beta_{j k} \equiv \mathbf{E} \psi_{j k}\left(X_{\ell}\right)\right),
\end{aligned}
$$

последовательности коэффициентов, соответствующие проекциям векторов $\boldsymbol{\xi}_{\ell}$ и $\mathbf{E} \xi_{\ell}$ на отдельные уровни разрешения. Все эти последовательности принадлежат $l^{2}(\mathbf{Z}) \cap l^{p}(\mathbf{Z})$, а случайные векторы $\xi_{\ell}^{(j)} \in l^{p}(\mathbf{Z})$, $j=J_{*}, \ldots, J^{*}$, ограничены с вероятностью 1. Действительно, по условию (1.7) никакая точка из $\mathbf{R}$ не может принадлежать носителям более чем $2 K-1$ базисных функций одного уровня разрешения $j$, поэтому

$$
\forall x \quad \sum_{k}\left|\phi_{J_{*} k}(x)\right|^{p} \leqslant 2 K \Phi_{1}^{p} \lambda_{J_{*}}^{-p / 2}, \quad \sum_{k}\left|\psi_{j k}(x)\right|^{p} \leqslant 2 K \Phi_{1}^{p} \lambda_{j}^{-p / 2} .
$$

Следовательно, $\quad\left|\xi^{(j)}\right|_{p} \leqslant(2 K)^{1 / p} \Phi_{1} \lambda_{j}^{-1 / 2}$ и $\left\|\left(\mathscr{K}_{j}-\mathscr{K}_{j-1}\right) \boldsymbol{\xi}\right\|_{p} \leqslant$ $c_{2}(2 K)^{1 / p} \Phi_{1} \lambda_{j}^{-(1-1 / p)}$ (см. (1.9)). В соединении с равенством $\lambda_{j+1}^{-1}=2 \lambda_{j}^{-1}$ приведенные оценки показывают, что

$$
\begin{aligned}
\left\|\xi_{\ell}\right\|_{p} & =\left\|\sum_{k} \phi_{J_{*} k}\left(X_{\ell}\right) \phi_{J_{*} k}+\sum_{J_{*}<j \leqslant J^{*}} \sum_{k} \psi_{j k}\left(X_{\ell}\right) \psi_{j k}\right\|_{p} \\
& \leqslant c_{2}(2 K)^{1 / p} \Phi_{1}\left(\lambda_{J_{*}}^{-(1-1 / p)}+\sum_{J_{*}<j \leqslant J^{*}} \lambda_{j}^{-(1-1 / p)}\right) \leqslant \frac{1}{2} H,
\end{aligned}
$$

где $H=C_{1}^{*} K^{1 / p} \Phi_{1} \lambda_{J^{*}}^{-(1-1 / p)}$, а константа $C_{1}^{*}=c_{2} 2^{1+1 / p}\left(1-2^{-(1-1 / p)}\right)$ зависит лишь от $p$.

Мы будем сравнивать значения ВОП $\tilde{f}$, определенной в (1.4), со значениями $\hat{f}=\hat{f}_{n}$ из (1.5). Как $\tilde{f}$, так и $\hat{f}$ задаются конечными линейными комбинациями базисных всплесков и могут рассматриваться как случайные векторы в $L^{p}(\mathbf{R})$.

Случайный вектор $\hat{f}$ является проекцией выборочного среднего (2.2) на $V_{J_{*}} \oplus H_{J^{*}}^{\gamma \delta}$, получаюшейся исключением компонент $\overline{\boldsymbol{\xi}}$ с малыми математическими ожиданиями, а его математическое ожидание - соответствуюшая проекция плотности $f$. Во введенных выше обозначениях второе слагаемое правой части (1.6) есть $\hat{f}-\mathbf{E} \hat{f}=\left(\mathscr{K}_{J_{*}}+\mathscr{K}_{J^{*}}^{\gamma \delta}\right)(\overline{\boldsymbol{\xi}}-\mathbf{E} \overline{\boldsymbol{\xi}})$. 
Известно [10], что при $p \in[2, \infty)$ банаховы пространства $\mathscr{X}=L^{p}, l^{p}$ принадлежат к типу $\mathscr{G}$. Именно, существует отображение $g: \mathscr{X} \rightarrow \mathscr{X}^{*}$ такое, что $\|g(x)\|_{p^{\prime}}=\|x\|_{p}, p^{\prime}=1 /(1-1 / p)$, и $\langle x, g(x)\rangle=\|x\|_{p}^{2}, \| g(x)-$ $g(y)\left\|_{p^{\prime}} \leqslant G\right\| x-y \|_{p}$ для любых $x, y \in \mathscr{X}$. В банаховом пространстве типа $\mathscr{G}$

$$
\mathbf{E}\left\|\xi_{1}+\cdots+\xi_{n}\right\|^{2} \leqslant G \sum_{\ell=1}^{n} \mathbf{E}\left\|\xi_{\ell}\right\|^{2},
$$

если случайные векторы $\xi_{\ell}, \ell=1, \ldots, n$, независимы и центрированы, $\mathbf{E} \xi_{\ell}=0$. (При $\mathscr{X}=L^{p}, l^{p}$ константа выписывается явно: $G=2 p-3$.)

Д ок а з а те л ь с т в о т е о р е м ы 1.2. Вследствие (2.7) справедливо неравенство $\mathbf{E}\|\hat{f}-\mathbf{E} \hat{f}\|_{p} \leqslant\left(\mathbf{E}\|\hat{f}-\mathbf{E} \hat{f}\|_{p}^{2}\right)^{1 / 2} \leqslant n^{-1} \tau_{0} B$, где $\tau_{0}=\sqrt{G}$ и $B^{2}=n \mathbf{V}_{\gamma \delta}$. Поскольку $\left\|\xi_{\ell}-\mathbf{E} \xi_{\ell}\right\|_{p} \leqslant H$ в соответствии с (2.6), теорема следует из известного неравенства для сумм независимых векторов в банаховом пространстве [16, теорема 3.3.1].

2.2. Оценка невязки с использованием мягкой срезки. При оценке слагаемого $\|\tilde{f}-\hat{f}\|_{p}$ в (1.6) оказывается удобным использовать кратномасштабные разложения с коэффициентами, полученнєіми применением «мягкой срезки»

$$
\left|b_{j k}-\beta_{j k}\right|_{\gamma \delta_{j}}=\max \left\{\left|b_{j k}-\beta_{j k}\right|-\gamma \delta_{j}, 0\right\},
$$

где функция $|x|_{\alpha}=\max \{|x|-\alpha, 0\}, \alpha>0$, выпукла. Следующая лемма представляет собой адаптацию предложения 10.3 книги [12]. Выкладки приведены для удобства читателя.

Лемма 2.1. Для любого $\gamma \in\left(0, \frac{1}{4}\right)$ проекиия разности $\tilde{f}-\hat{f}$ на отдельный уровень разрешения допускает оченку

$$
\left\|\left(\mathscr{K}_{j}-\mathscr{K}_{j-1}\right)(\tilde{f}-\hat{f})\right\|_{p} \leqslant c_{2}\left(\gamma^{-1} \mathbf{w}_{j}+(1+4 \gamma) \mathbf{b}_{j}\left(\gamma, \delta_{j}\right)\right) \lambda_{j}^{-(1 / 2-1 / p)},
$$

где $\mathbf{w}_{j}=\left(\sum_{k}\left|b_{j k}-\beta_{j k}\right|_{\gamma \delta_{j}}^{p}\right)^{1 / p}$, константа $c_{2}-$ та же, что в (1.9), а величина $\mathrm{b}_{j}\left(\gamma, \delta_{j}\right)$ определена в (1.11). Кроме того, для $\mathbf{W}=$ $\sum_{J_{*}<j \leqslant J^{*}} \lambda_{j}^{-(1 / 2-1 / p)} \mathbf{w}_{j}$ u $\mathbf{B}(\gamma, \delta)$ формульл (1.10) вьгполняется неравенство

$$
\|\tilde{f}-\hat{f}\|_{p} \leqslant c_{2} \gamma^{-1} \mathbf{W}+c_{2}(1+4 \gamma) \mathbf{B}(\gamma, \delta) .
$$

Д ок а з а т ел ь с т в о. Определим множество $K_{j}^{\gamma}=\left\{k:\left|\beta_{j k}\right|<\right.$ $\left.(1+2 \gamma) \delta_{j}\right\}$ и обозначим $\Delta_{j k}=\widetilde{b}_{j k}-b_{j k} 1_{\left\{\left|\beta_{j k}\right| \geqslant(1+2 \gamma) \delta_{j}\right\}}$ отдельную координату кратномасштабного разложения вектора $\mathscr{K}_{j}(\tilde{f}-\hat{f})$. Легко видеть, что $\left|\Delta_{j k}\right|=\left|b_{j k}\right| 1_{\left\{\left|b_{j k}\right| \geqslant \delta_{j}\right\}}$, если $k \in K_{j}^{\gamma}$, и $\left|\Delta_{j k}\right|=\left|b_{j k}\right| 1_{\left\{\left|b_{j k}\right|<\delta_{j}\right\}}$, если $k \notin K_{j}^{\gamma}$, так что вследствие (1.9)

$$
\left\|\left(\mathscr{K}_{j}-\mathscr{K}_{j-1}\right)(\tilde{f}-\hat{f})\right\|_{p} \leqslant c_{2} \lambda_{j}^{-(1 / 2-1 / p)}\left(S_{1}+S_{2}\right)^{1 / p},
$$

где $S_{1}=\sum_{k \in K_{j}^{\gamma}}\left|b_{j k}\right|^{p} 1_{\left\{\left|b_{j k}\right| \geqslant \delta_{j}\right\}}$ и $S_{2}=\sum_{k \notin K_{j}^{\gamma}}\left|b_{j k}\right|^{p} 1_{\left\{\left|b_{j k}\right|<\delta_{j}\right\}}$. 
Чтобы облегчить выкладки, воспользуемся на время сокрашениями $b=b_{j k}, \beta=\beta_{j k}$ и $\gamma \delta=\gamma \delta_{j}$.

Рассмотрим сначала одно слагаемое суммы $S_{1}$, для которого выполняются неравенства $|b|>\delta$ и $|\beta| \leqslant(1+2 \gamma) \delta$.

Если $|b-\beta|<2 \gamma \delta$, то $|b| \leqslant|\beta|+2 \gamma \delta \leqslant|\beta|+2 \gamma|b|$, поэтому $|b| \leqslant(1-2 \gamma)^{-1}|\beta| \leqslant(1+4 \gamma)|\beta|$.

Если $|b-\beta| \geqslant 2 \gamma \delta$, то $|b| \leqslant|\beta|+|b-\beta| \leqslant|\beta|+2|b-\beta|_{\gamma \delta}$, поскольку $|b-\beta| \leqslant \gamma \delta+|b-\beta|_{\gamma \delta}$.

Результатом приведенного рассуждения оказывается оценка $\left|\Delta_{j k}\right| \leqslant(1+$ $4 \gamma)\left|\beta_{j k}\right|+2\left|b_{j k}-\beta_{j k}\right|_{\gamma \delta_{j}}$ для $k \in K_{j}^{\gamma}$.

Для слагаемых $S_{2}$ ограничения на $b$ и $\beta$ суть $|b| \leqslant \delta$ и $|\beta| \geqslant(1+2 \gamma) \delta$. В этом случае $|b-\beta| \geqslant 2 \gamma \delta \geqslant 2 \gamma|b|$, а потому

$$
\left|\Delta_{j k}\right| \leqslant \frac{1}{2 \gamma}\left|b_{j k}-\beta_{j k}\right| 1_{\left\{\left|b_{j k}-\beta_{j k}\right| \geqslant 2 \gamma \delta_{j}\right\}} \leqslant \frac{1}{\gamma}\left|b_{j k}-\beta_{j k}\right|_{\gamma \delta}, \quad k \notin K_{j}^{\gamma} .
$$

Используя то обстоятельство, что $S_{1}^{1 / p}$ и $S_{2}^{1 / p}$ являются $l^{p}$-нормами подпоследовательностей координат вектора из $l^{p}(\mathbf{Z})$, занумерованных числами из непересекаюшихся подмножеств Z, мы можем сделать заключение о справедливости неравенства

$$
\left(S_{1}+S_{2}\right)^{1 / p} \leqslant \max \left\{2, \frac{1}{\gamma}\right\}\left(\sum_{k \in \mathbf{Z}}\left|b_{j k}-\beta_{j k}\right|_{\gamma \delta_{j}}^{p}\right)^{1 / p}+(1+4 \gamma)\left(\sum_{k \in K_{j}^{\gamma}}\left|\beta_{j k}\right|^{p}\right)^{1 / p}
$$

которое влечет первую из оценок леммы. Оценка (2.10) получается применением неравенства треугольника в пространстве $l^{p}$.

\section{3. Производящие функции}

3.1. Рандомизация объема выборки. Целью этого пункта является вывод оценок для производящей функции случайной величины $\mathbf{w}_{j}$ из (2.9). Для гауссовского случайного вектора с тем же ковариационным оператором, что $\xi_{1}$, все координаты кратномасштабного разложения слабо коррелированы и, следовательно, почти независимы. Выборочные средние $a_{J_{*} k}=\overline{\phi_{J_{*} k}(X)}$ и $b_{j k}=\overline{\psi_{j k}(X)}$ обладают сходными свойствами. Для использования того, что они почти независимы, удобно применить рандомизацию объема выборки (этот подход использовался, например, при исследовании асимптотического поведения ядерных оценок функции регрессии [4] и плотности [15] двумерных распределений).

Рассмотрим бесконечную последовательность $\left(X_{\ell}, \ell \in \mathbf{N}\right)$ независимых одинаково распределенных наблюдений с плотностью распределения $f$ из (1.1) и целочисленную случайную величину

$$
N: \Omega \rightarrow \mathbf{Z}_{+}
$$


распределенную по закону Пуассона со средним $\mathbf{E} N=n$ и независимую от $\left(X_{\ell}, \ell \in \mathbf{N}\right)$. Известно (см. [13, § 1.2] или $\left.[3, \S 1.5]\right)$, что случайная мера, заданная на борелевских множествах равенством

$$
\nu(B)=\sum_{0<\ell \leqslant N} 1\left\{X_{\ell} \in B\right\}, \quad B \in \mathscr{B}(\mathbf{R}),
$$

определяет в $\mathbf{R}$ пуассоновский точечный процесс с мерой интенсивности $\mu(B)=n \int_{B} f(x) d x$. Для ограниченной измеримой функции $g: \mathbf{R} \rightarrow \mathbf{R}$, обладающей компактным носителем, значения характеристического функционала и функционала Лапласа точечного процесса $\nu$ выражаются формулой (где, соответственно, $z=i$ или $z=1$ )

$$
\begin{aligned}
& \Phi(z g)=\mathbf{E} \exp \{z\langle g, \nu\rangle\}=\exp \left\{n \int\left(e^{z g(x)}-1\right) f(x) d x\right\}, \\
& \langle g, \nu\rangle=\int g(x) d \nu(x)=\sum_{0<\ell \leqslant N} g\left(X_{\ell}\right) .
\end{aligned}
$$

Из (3.2) немедленно следует, что случайные величины $\left\langle g_{k}, \nu\right\rangle, k=$ $1, \ldots, m$, независимы, если носители функций $g_{\ell}: \mathbf{R} \rightarrow \mathbf{R}$ не пересекаются, - действительно, в этом случае

$$
\mathbf{E} \exp \left\{i \sum_{k=1}^{m} \tau_{k}\left\langle g_{k}, \nu\right\rangle\right\}=\prod_{k=1}^{m} \mathbf{E} \exp \left\{i \tau_{k}\left\langle g_{k}, \nu\right\rangle\right\},
$$

поскольку $\exp \left\{i \sum_{k=1}^{m} \tau_{k} g_{k}(x)\right\}-1=\sum_{k=1}^{m}\left(\exp \left\{i \tau_{k} g_{k}(x)\right\}-1\right)$.

Воспользуемся обозначениями (3.2), чтобы определить

$$
\begin{aligned}
\mathbf{u}_{j, s}^{p} & =\sum_{k}\left|\frac{1}{n}\left\langle\psi_{j(s+K k)}, \nu\right\rangle-\beta_{j(s+K k)}\right|_{\gamma \delta_{j}}^{p}, \\
\mathbf{u}_{j}^{p} & =\sum_{k}\left|\frac{1}{n}\left\langle\psi_{j k}, \nu\right\rangle-\beta_{j k}\right|_{\gamma \delta_{j}}^{p} .
\end{aligned}
$$

Лемма 3.1. При всяком $h>0$ суммы в формулах $(2.9),(2.10)$ удовлетворяют неравенству

$$
\mathbf{E} \exp \{h \mathbf{W}\} \leqslant \frac{8}{3} \exp \left\{\frac{2 h}{\sqrt{n}} \mathbf{B}^{+}\right\} \max _{J_{*}<j \leqslant J^{*}} \mathbf{E} \exp \left\{\widehat{h} \mathbf{u}_{j}\right\}
$$

где $\widehat{h}=h \lambda_{J^{*}}^{-(1 / 2-1 / p)} \chi(p)$, а величина $\chi(p)$ та же, что в теореме 1.1 . Кроме того,

$$
\mathbf{E} \exp \left\{\widehat{h} \mathbf{u}_{j}\right\} \leqslant \max _{0 \leqslant s<K} \mathbf{E} \exp \left\{\widehat{h} K^{1 / p} \mathbf{u}_{j, s}\right\}
$$

3 а м е ч а н и е 3.1. Заметим, что в (3.3) случайные величины $n^{-1}\left\langle\psi_{j(s+K k)}, \nu\right\rangle-\beta_{j(s+K k)}, k \in \mathbf{Z}$, независимы, поскольку носители функций $\psi_{j(s+K k)}$ не имеют общих точек. 
Доказательст во лем мы 3.1. Определим для $j \in \mathbf{N} \cap$ $\left(J_{*}, J^{*}\right]$ и $k \in \mathbf{Z}$ последовательность

$$
Z_{0, j k}=0, \quad Z_{t, j k}=Z_{t-1, j k}+\left(\psi_{j k}\left(X_{t}\right)-\beta_{j k}\right), \quad t=1,2, \ldots .
$$

Для каждой пары $(j, k)$ последовательность $Z_{t, j k}, t \in \mathbf{Z}_{+}$, является мартингалом по отношению к потоку $\sigma$-алгебр $\mathscr{F}_{t}=\sigma\left(X_{\ell}, \ell \leqslant t\right)$ вследствие независимости $X_{\ell}$. Функция $\left|n^{-1} x\right|_{\gamma \delta_{j}}$ выпукла, а потому для каждой пары $(j, k)$ последовательность $t \mapsto\left|n^{-1} Z_{t, j k}\right|_{\gamma \delta_{j}}$ образует субмартингал. Последовательности (ниже $t=0,1,2 \ldots$ )

$$
\mathbf{Z}(t)=\sum_{J_{*}<j \leqslant J^{*}} \lambda_{j}^{1 / 2-1 / p}\left(\sum_{k}\left|\frac{1}{n} Z_{t, j k}\right|_{\gamma \delta_{j}}^{p}\right)^{1 / p}, \quad \exp \{h \mathbf{Z}(t)\}
$$

также являются субмартингалами благодаря выпуклости нормы $l^{p}$ и показательной функции.

По построению $\left|n^{-1} Z_{n, j k}\right|_{\gamma \delta_{j}}=\left|b_{j k}-\beta_{j k}\right|_{\gamma \delta_{j}}$ и $\mathbf{Z}(n)=\mathbf{W}$, а потому

$$
\mathbf{E} \exp \{h \mathbf{W}\} \leqslant \mathbf{E} \exp \{h \mathbf{Z}(t)\}, \quad t \geqslant n .
$$

Для выборки случайного объема (3.1) из независимости $N$ и $\left(X_{\ell}, \ell \in \mathbf{N}\right)$ следует, что

$$
\begin{aligned}
\mathbf{E} \exp \{h \mathbf{W}\} & =\mathbf{E} \exp \{h \mathbf{Z}(n)\} \leqslant \sum_{n \leqslant t \leqslant n+2 \sqrt{n}} \mathbf{E} \exp \{h \mathbf{Z}(t)\} \frac{\mathbf{P}\{N=t\}}{\mathbf{p}} \\
& \leqslant \frac{8}{3} \mathbf{E} \exp \{h \mathbf{Z}(N)\} 1\{0 \leqslant N-n \leqslant 2 \sqrt{n}\},
\end{aligned}
$$

где $\mathbf{p}=\mathbf{P}\{n \leqslant N \leqslant n+2 \sqrt{n}\} \geqslant \frac{3}{8}$. Оценка для $\mathbf{p}$ вытекает из неравенства Чебышёва в форме $\mathbf{P}\{|N-\mathbf{E} N| \leqslant 2 \sqrt{n}\} \geqslant \frac{3}{4}$. Действительно,

$$
\mathbf{p} \geqslant 1-\mathbf{P}\{|N-\mathbf{E} N|>2 \sqrt{n}\}-\mathbf{q} \geqslant \frac{3}{4}-\mathbf{q},
$$

где $\mathbf{q}=\sum_{k=1}^{M} \mathbf{P}\{N=n-k\}$ и $M=[2 \sqrt{n}]$. Отсюда можно заключить, что $2 \mathbf{p} \geqslant \frac{3}{4}$, поскольку

$$
\begin{aligned}
\mathbf{q} & =\sum_{k=1}^{M} \frac{n^{n-k} e^{-n}}{(n-k) !}=\sum_{k=0}^{M-1} \frac{n^{n+k} e^{-n}}{(n+k) !} \frac{(n+k) ! n^{-(2 k+1)}}{(n-k-1) !} \\
& =\sum_{k=0}^{M-1} \frac{n^{n+k} e^{-n}}{(n+k) !} \prod_{\ell=1}^{k}\left(1-\frac{\ell^{2}}{n^{2}}\right) \leqslant \mathbf{p}=\sum_{k=0}^{M} \frac{n^{n+k} e^{-n}}{(n+k) !} .
\end{aligned}
$$

Легко видеть, что $|a+b|_{t} \leqslant|a|_{t}+|b|, a, b \in \mathbf{R}, t>0$, для мягкой срезки (2.8). Пользуясь обозначениями (3.2), можно представить коэффициенты многоуровневого разложения (3.6) при $t=N$ в виде

$$
\left|\frac{1}{n} Z_{N, j k}\right|_{\gamma \delta_{j}}=\left|\frac{1}{n}\left\langle\psi_{j k}, \nu\right\rangle-\frac{N}{n} \beta_{j k}\right|_{\gamma \delta_{j}} \leqslant\left|\frac{1}{n}\left\langle\psi_{j k}, \nu\right\rangle-\beta_{j k}\right|_{\gamma \delta_{j}}+\left|\frac{N-n}{n} \beta_{j k}\right| .
$$


Из (1.12) и неравенства треугольника в $l^{p}$ следует, что при $n \leqslant N \leqslant$ $n+2 \sqrt{n}$

$$
\begin{aligned}
\mathbf{Z}(N) & =\sum_{J_{*}<j \leqslant J^{*}} \lambda_{j}^{-(1 / 2-1 / p)}\left(\sum_{k}\left|\frac{1}{n}\left\langle\psi_{j k}, \nu\right\rangle-\frac{N}{n} \beta_{j k}\right|_{\gamma \delta_{j}}^{p}\right)^{1 / p} \\
& \leqslant \sum_{J_{*}<j \leqslant J^{*}} \lambda_{j}^{-(1 / 2-1 / p)}\left(\mathbf{u}_{j}+\frac{N-n}{n} \mathbf{b}_{j}^{+}\right) \\
& \leqslant \sum_{J_{*}<j \leqslant J^{*}} \lambda_{j}^{-(1 / 2-1 / p)}\left(\mathbf{u}_{j}+\frac{2}{\sqrt{n}} \mathbf{b}_{j}^{+}\right) .
\end{aligned}
$$

Мы заключаем отсюда, что при $h>0$ и $\mathbf{U}=\sum_{J_{*}<j \leqslant J^{*}} \lambda_{j}^{-(1 / 2-1 / p)} \mathbf{u}_{j}$

$$
\begin{aligned}
\mathbf{E} \exp \{h \mathbf{W}\} & \leqslant \frac{8}{3} \mathbf{E} \exp \{h \mathbf{Z}(N)\} 1\{0 \leqslant N-n \leqslant 2 \sqrt{n}\} \\
& \leqslant \frac{8}{3} \exp \left\{\frac{2 h}{\sqrt{n}} \mathbf{B}^{+}\right\} \mathbf{E} \exp \{h \mathbf{U}\} .
\end{aligned}
$$

Чтобы закончить доказательство (3.4), представим величину $\mathbf{U}$ в правой части (3.7) в виде

$$
\mathbf{U}=\lambda_{J^{*}}^{-(1 / 2-1 / p)} \chi(p) \sum_{J_{*}<j \leqslant J^{*}} \alpha_{j} \mathbf{u}_{j}, \quad \sum_{J_{*}<j \leqslant J^{*}} \alpha_{j}=1,
$$

с $\alpha_{j}=\left(\lambda_{J^{*}} / \lambda_{j}\right)^{1 / 2-1 / p} / \chi(p)=2^{-(1 / 2-1 / p)\left(J^{*}-j\right)} / \chi(p)$ и воспользуемся выпуклостью показательной функции, чтобы придти к неравенству

$$
\mathbf{E} \exp \left\{h \sum_{J_{*}<j \leqslant J^{*}} \lambda_{j}^{-(1 / 2-1 / p)} \mathbf{u}_{j}\right\} \leqslant \sum_{J_{*}<j \leqslant J^{*}} \alpha_{j} \mathbf{E} \exp \left\{\widehat{h} \mathbf{u}_{j}\right\} .
$$

Неравенство (3.5) следует из выпуклости функции $\exp \left\{\widehat{h}|\mathbf{x}|_{p}\right\}, \mathbf{x} \in l^{p}$ : $\mathbf{E} \exp \left\{\widehat{h} \mathbf{u}_{j}\right\}=\mathbf{E} \exp \left\{\widehat{h} K^{1 / p}\left(\frac{1}{K} \sum_{s=0}^{K-1} \mathbf{u}_{j, s}^{p}\right)^{1 / p}\right\} \leqslant \frac{1}{K} \sum_{s=0}^{K-1} \mathbf{E} \exp \left\{\widehat{h} K^{1 / p} \mathbf{u}_{j, s}\right\}$.

3.2. Производящие функции «редких» сумм. Мы выведем сначала оценку для производящей функции отдельной координаты разложения (3.3) из формулы (3.2), описывающей функционал Лапласа для пуассоновского точечного процесса $\nu$.

Лемма 3.2. Если вьполненьл условия (1.7), то при $h>0$

$$
\begin{aligned}
& \mathbf{E} \exp \left\{h\left|\frac{1}{n}\left\langle\psi_{j k}, \nu\right\rangle-\beta_{j k}\right|_{\gamma \delta_{j}}\right\} \\
& \leqslant 1+\frac{2 h \mathbf{v}_{j k} \mathscr{E}_{j}^{2}(h)}{\gamma \delta_{j} n} \exp \left\{-h \gamma \delta_{j}+\frac{h^{2}}{n}\left(1+\frac{1}{2} \mathscr{E}_{j}(h)\right) \mathbf{v}_{j k}\right\},
\end{aligned}
$$

где $\mathscr{E}_{j}(h)=\exp \left\{\Phi_{1} h \lambda_{j}^{-1 / 2} n^{-1}\right\} u \mathbf{v}_{j k}=\int_{\mathbf{R}} \psi_{j k}^{2}(x) f(x) d x$.

Заметим, что $\mathscr{E}_{j}(h) \leqslant \mathscr{E}_{J^{*}}(h)$, если $j \leqslant J^{*}$. Оценка производящей функции моментов «редкой» суммы $\mathbf{u}_{j, s}$ приведена в следующей лемме. 
Лемма 3.3. $\quad$ В обозначениях леммы 3.1 и леммы 3.2

$\mathbf{E} \exp \left\{h \mathbf{u}_{j, s}\right\} \leqslant \exp \left\{\frac{2 h \overline{\mathbf{v}}_{j} \mathscr{E}_{j}^{2}(h)}{\gamma \delta_{j} n \lambda_{j}} \exp \left\{-h \gamma \delta_{j}+\frac{h^{2}}{n}\left(1+\frac{1}{2} \mathscr{E}_{j}(h)\right) \mathbf{v}_{j}^{*}\right\}\right\}$, если $\max _{\ell} \mathbf{v}_{j \ell} \leqslant \mathbf{v}_{j}^{*} u \lambda_{j} \max _{s} \sum_{k} \mathbf{v}_{j(s+K k)} \leqslant \overline{\mathbf{v}}_{j}(c \mathcal{M} .(1.13))$.

Д ок а з тель с тво ле м мы 3.3. Применим грубую верхнюю оценку $|\eta|_{p} \leqslant|\eta|_{1}$ в форме

$$
\mathbf{u}_{j, s} \leqslant \sum_{k}\left|\frac{1}{n}\left\langle\psi_{j(s+K k)}, \nu\right\rangle-\beta_{j(s+K k)}\right|_{\gamma \delta_{j}}
$$

и воспользуемся независимостью слагаемых в правой части (см. замечание 3.1 ), чтобы придти к неравенству

$$
\mathbf{E} \exp \left\{h \mathbf{u}_{j, s}\right\} \leqslant \prod_{k} \mathbf{E} \exp \left\{h\left|\frac{1}{n}\left\langle\psi_{j(s+K k)}, \nu\right\rangle-\beta_{j(s+K k)}\right|_{\gamma \delta_{j}}\right\},
$$

справедливому при всяком $s$ и $h>0$. Неравенство леммы вытекает теперь из оценки леммы 3.2 и неравенства $1+x \leqslant e^{x}$.

Д ок а затель с т в о л е м м ы 3.2. Ниже мы пользуемся сокрашениями $\psi=\psi_{j k}, \zeta=n^{-1}\left\langle\psi_{j k}, \nu\right\rangle-\beta_{j k}, \delta=\delta_{j}, \mathbf{v}=\mathbf{v}_{j k}$ и $A_{+}=\left\{|\zeta|>\gamma \delta_{j}\right\}$. Легко видеть, что при $h \geqslant 0$

$$
\left|\exp \left\{h|\zeta|_{\gamma \delta}\right\}-1\right| \leqslant e^{-h \gamma \delta} 1_{A_{+}}|\exp \{h|\zeta|\}-1| \leqslant e^{-h \gamma \delta} \frac{|\zeta|}{\gamma \delta}|\exp \{h|\zeta|\}-1| \text {. }
$$

Соединяя приведенное выше неравенство с оценками $\left|e^{x}-1\right| \leqslant|x| e^{|x|}$ и $\exp \{|x|\} \leqslant 2 \operatorname{ch} x$, заключаем, что

$$
\begin{aligned}
\mathbf{E} \exp \left\{h|\zeta|_{\gamma \delta}\right\} & \leqslant 1+\frac{h e^{-h \gamma \delta}}{\gamma \delta} \mathbf{E} \zeta^{2} \exp \{h|\zeta|\} \leqslant 1+\frac{2 h e^{-h \gamma \delta}}{\gamma \delta} \mathbf{E} \zeta^{2} \operatorname{ch}\{h \zeta\} \\
& =1+\frac{2 h e^{-h \gamma \delta}}{\gamma \delta} \frac{d^{2}}{d h^{2}} \mathbf{E} \operatorname{ch}\{h \zeta\} .
\end{aligned}
$$

Производящую функцию в правой части (3.9) можно выразить явно с помощью формулы (3.2) и равенства $\beta_{j k}=\mathbf{E}\left\langle\psi_{j k}, \nu\right\rangle=\int \psi(x) f(x) d x$ :

$$
\begin{aligned}
\mathbf{E} \operatorname{ch}\{h \zeta\} & =\frac{1}{2}(\mathbf{E} \exp \{h \zeta\}+\mathbf{E} \exp \{-h \zeta\}) \\
& =\frac{1}{2}\left(\exp \left\{n \Psi\left(\frac{1}{n} h\right)\right\}+\exp \left\{n \Psi\left(-\frac{1}{n} h\right)\right\}\right)
\end{aligned}
$$

где $\Psi(\tau)=\int(\exp \{\tau \psi(x)\}-1-\tau \psi(x)) f(x) d x$.

Формула Тейлора для показательной функции и неравенство $|\psi(x)| \leqslant \psi=\Phi_{1} \lambda_{j}^{-1 / 2}$, являюшееся следствием (1.2) и (1.7), доставляют для $\Psi$ и ее производных следующие оценки:

$$
\begin{aligned}
|\Psi( \pm \tau)| & \leqslant \frac{1}{2} \tau^{2} \int e^{\tau|\psi(x)|} \psi^{2}(x) f(x) d x \leqslant \frac{1}{2} \tau^{2} e^{\tau \psi} \mathbf{v} \\
\left|\Psi^{\prime}( \pm \tau)\right| & =\left| \pm \int \psi\left(e^{ \pm \tau \psi(x)}-1\right) f(x) d x\right| \leqslant \tau e^{\tau \psi} \mathbf{v} \\
\left|\Psi^{\prime \prime}( \pm \tau)\right| & =\left|\int \psi^{2} e^{ \pm \tau \psi(x)} f(x) d x\right| \leqslant e^{\tau \psi} \mathbf{v}
\end{aligned}
$$


Из явного выражения производной в (3.9) следует, что

$$
\begin{aligned}
\frac{d^{2}}{d h^{2}} \mathbf{E} \operatorname{ch}\{h \zeta\} & \leqslant \max _{\tau= \pm h / n}\left\{e^{n \Psi(\tau)}\left(\frac{1}{n} \Psi^{\prime \prime}(\tau)+\left(\Psi^{\prime}(\tau)\right)^{2}\right)\right\} \\
& \leqslant \exp \left\{\frac{1}{2 n} h^{2} e^{\psi h / n} \mathbf{v}\right\}\left(e^{\psi h / n}\right)^{2} \frac{1}{n} \mathbf{v}\left(1+\frac{1}{n} h^{2} \mathbf{v}\right) .
\end{aligned}
$$

Чтобы придти к неравенству леммы, остается соединить неравенство $1+n^{-1} h^{2} \mathbf{v} \leqslant \exp \left\{n^{-1} h^{2} \mathbf{v}\right\}$ с вышеприведенными оценками.

\section{4. Доказательство теоремы 1.1}

По неравенству (2.10) леммы 2.1 и показательному неравенству Маркова при $h>0$

$$
\mathbf{p}_{\varepsilon} \leqslant \mathbf{P}\left\{\mathbf{W} \geqslant \gamma \varepsilon+\frac{2}{\sqrt{n}} \mathbf{B}^{+}\right\} \leqslant \exp \left\{-h\left(\frac{2}{\sqrt{n}} \mathbf{B}^{+}+\gamma \varepsilon\right)\right\} \mathbf{E} \exp \{h \mathbf{W}\} .
$$

Лемма 3.1 приводит эту оценку к виду

$$
\begin{aligned}
\mathbf{p}_{\varepsilon} & \leqslant \frac{8}{3} \exp \{-h \gamma \varepsilon\} \max _{j} \mathbf{E} \exp \left\{\widehat{h} \mathbf{u}_{j}\right\} \\
& \leqslant \frac{8}{3} \exp \{-h \gamma \varepsilon\} \max _{j} \max _{s} \mathbf{E} \exp \left\{K^{1 / p} \widehat{h} \mathbf{u}_{j, s}\right\},
\end{aligned}
$$

где $\widehat{h}=h \lambda_{J^{*}}^{-(1 / 2-1 / p)} \chi(p)$.

Заменим теперь $h$ на $H=K^{1 / p} \widehat{h}$, а $\varepsilon$ на $\widehat{\varepsilon}=\gamma \varepsilon \lambda_{J^{*}}^{(1 / 2-1 / p)}\left(K^{1 / p} \chi(p)\right)^{-1}$. Используя лемму 3.3 и условие (1.13), приходим к оценке

$$
\mathbf{p}_{\varepsilon} \leqslant \frac{8}{3} \max _{J_{*}<j \leqslant J^{*}} \exp \{-H \widehat{\varepsilon}\} \exp \left\{\frac{2 H \overline{\mathbf{v}}^{*} \mathscr{E}_{j}^{2}(H)}{\varkappa \gamma \delta_{J^{*}} n \lambda_{J^{*}}} \exp \left\{U_{j}(H)\right\}\right\},
$$

где $\mathscr{E}_{j}(H)=\exp \left\{\Phi_{1} H \lambda_{j}^{-1 / 2} n^{-1}\right\} \leqslant \mathscr{E}_{J^{*}}(H)$ и по условию (1.13)

$$
\begin{aligned}
U_{j}(H) & =-H \gamma \delta_{j}+\frac{1}{n} H^{2}\left(1+\frac{1}{2} \mathscr{E}_{j}(H)\right) \mathbf{v}^{*} \\
& \leqslant H\left(-\varkappa \gamma \delta_{J^{*}}+\frac{1}{n} H\left(1+\frac{1}{2} \mathscr{E}_{J^{*}}(H)\right) \mathbf{v}^{*}\right) .
\end{aligned}
$$

Остается выбрать подходяшее значение параметра $H$, с тем чтобы удовлетворить сначала неравенству $\mathscr{E}_{J^{*}}(H) \leqslant 2$. Выбор, который используется ниже, есть $H=\left(4 \mathbf{v}^{*}\right)^{-1} \varkappa \gamma \delta_{J^{*}} n$. При условии (1.14) он гарантирует выполнение неравенств $\mathscr{E}_{J^{*}}(H) \leqslant 2$ и

$$
U_{j}(H) \leqslant H\left(-\varkappa \gamma \delta_{J^{*}}+\frac{2 \mathbf{v}^{*}}{n} H\right)=-\frac{\left(\varkappa \gamma \delta_{J^{*}} \sqrt{n}\right)^{2}}{8 \mathbf{v}^{*}} .
$$

Эти последние приводят к оценке теоремы:

$$
\begin{aligned}
\mathbf{p} & \leqslant \frac{8}{3} \exp \{-H \widehat{\varepsilon}\} \exp \left\{\frac{8 H \overline{\mathbf{v}}^{*}}{\varkappa \gamma \delta_{J^{*}} n \lambda_{J^{*}}} \exp \left\{-\frac{\left(\varkappa \gamma \delta_{J^{*}} \sqrt{n}\right)^{2}}{8 \mathbf{v}^{*}}\right\}\right\} \\
& =\frac{8}{3} \exp \left\{-\frac{\varkappa \gamma \delta_{J^{*}} n \varepsilon \lambda_{J^{*}}^{(1 / 2-1 / p)}}{4 K^{1 / p} \chi(p) \mathbf{v}^{*}}\left(1-\frac{8 K^{1 / p} \chi(p) \overline{\mathbf{v}}^{*}}{\varepsilon \lambda_{J^{*}}^{3 / 2-1 / p} \sqrt{n}} \mu_{n}\right)\right\} .
\end{aligned}
$$


Авторы искренне признательны рецензенту за тщательное чтение рукописи и в особенности за указание примеров использования рандомизации объема выборки в работах [4], [15] и кандидатской диссертации В. В. Статулявичюса (Вильнюс, 1986).

\section{СПИСОК ЛИТЕРАТУРЫ}

1. Бернштейн С. Н. Теория вероятностей. М.-Л.: Гостехиздат, 1946, 556 с. $^{5 \text { ) }}$

2. Деврой Л., Дьёрфи Л. Непараметрическое оценивание плотности. $L_{1}$-подход. М.: Мир, 1988, 407 с.

3. Керстан Й., Маттес К., Мекке Й. Безгранично делимые точечные процессы. М.: Наука, 1982, 391 с.

4. Надарая Э. А. Предельное распределение квадратического уклонения непараметрических оценок функции регрессии. - Сообщ. АН ГССР, 1974, т. 74, № 1 , c. $33-36$.

5. Новиков И.Я., Стечкин С.Б. Основы теории всплесков. - Успехи матем. наук, 1998 , т. 53, № 6, с. 53-128.

6. Ченцов H.H. Оценка неизвестной плотности распределения по наблюдениям. Докл. АН СССР, 1962, т. 147, № 1, с. 45-48.

7. Daubechies I. Ten Lectures on Wavelets. Philadelphia: SIAM, 1992, 357 p.

8. Donoho D. L., Johnstone I. M., Kerkyacharian G., Picard D. Density estimation by wavelet thresholding. - Ann. Statist., 1996, v. 24, № 2, p. 508-539.

9. Fan J., Hall P., Martin M., Patil P. Adaptation to high spatial inhomogeneity using wavelet methods. - Statist. Sinica, 1999, v. 9, № 1, p. 85-102.

10. Fortet R., Mourier $E$. Les fonctions aléatoires comme éléments aléatoires dans les espaces de Banach. - Studia Math., 1955, v. 15, № 1, p. 62-79.

11. Hall P., McKay I., Turlach B. A. Performance of wavelet methods for functions with many discontinuities. - Ann. Statist., 1996, v. 24, № 6, p. 2462-2476.

12. Härdle W., Kerkyacharian G., Picard D., Tsybakov A. Wavelets, Approximation, and Statistical Applications. New York: Springer-Verlag, 1998, 265 p. (Lecture Notes in Statist., v. 129.)

13. Karr A.F. Point Processes and Their Statistical Inference. New York: Dekker, 1986, 490 p. (Probab. Pure Appl., v. 2.)

14. Meyer Y. Ondelettes et Opérateurs. V. I-III. Paris: Hermann, 1990-1991, 538 p.

15. Rosenblatt $M$. A quadratic measure of deviation of two-dimensional density estimates and a test of independence. - Ann. Statist., 1975, v. 3, № 1, p. 1-14.

16. Yurinsky V. Sums and Gaussian Vectors. Berlin: Springer-Verlag, 1995, 305 p. (Lecture Notes in Math., v. 1617.)

Поступила в редакцию

5.IX.2005

5) См. также воспроизведение исходной публикации: Бернштейн С. Н. Об одном видоизменении неравенства́ Чебышёва и о погрешности формулы Лапласа. - Собр. соч., т. 4. М.: Наука, 1964, с. 71-79. 